\title{
Prevalence of Perinatal Mortality and Associated Factors Among Neonate Admitted to Neonatal Intensive Care Unit of Jimma Medical Center, Oromia Region, Ethiopia
}

\author{
Abiru Neme*, Gadisa Bekele, Gebeye Muleta \\ Institute of Health, School of Nursing and Midwifery, Jimma University, Oromia, Ethiopia \\ Email address: \\ abiruneme@gmail.com (A. Neme) \\ ${ }^{*}$ Corresponding author
}

\section{To cite this article:}

Abiru Neme, Gadisa Bekele, Gebeye Muleta. Prevalence of Perinatal Mortality and Associated Factors Among Neonate Admitted to Neonatal Intensive Care Unit of Jimma Medical Center, Oromia Region, Ethiopia. International Journal of Clinical and Experimental Medical Sciences. Vol. 6, No. 4, 2020, pp. 71-78. doi: 10.11648/j.ijcems.20200604.14

Received: May 20, 2020; Accepted: June 1, 2020; Published: August 18, 2020

\begin{abstract}
Background: Death of an infant in utero or at birth has been always a devastating experience for the mother and of the concern in clinical practice. Infant mortality remains a challenge in the care of pregnant women worldwide, but particularly for developing countries and the need to understand contributory factors is crucial for addressing appropriate perinatal health. Objective: To assess perinatal mortality and associated factors among neonate admitted to neonatal intensive care unit of Jimma medical center, Jimma zone, Oromia region, Southwest Ethiopia, 2019. Method: One year retrospective study was conducted from April 10 to April 25, 2019 among neonate admitted to neonatal intensive care unit of Jimma Medical center. All neonate admitted to neonatal intensive care unit from April 10 to April 25, 2019 was included in the study. Descriptive statistics were used to analyses data by using SPSS version 21. The relationship between perinatal death and fetal or neonatal characteristics were analyzed. Significance association was made when $p<0.05$. Results: There was 191 total neonates admitted and 186 were included in the study period. The rate of perinatal death was $29.6 \%$. The majority $156(83.9 \%)$ of mother mode of delivery were spontaneous vaginal delivery. The dominant cause of admission to NICU was Sepsis followed by low birth weight and prematurity while the least was Perinatal Asphyxia and congenital malformation. The common cause of death among those admitted to NICU was prematurity, sepsis which accounts $67.9 \%$ and $20.75 \%$ respectively. Conclusions: This study showed that the magnitude of perinatal death at Jimma University Medical Centre is high. Most of babies were dying because of prematurity and sepsis. Improving quality of antenatal care and increasing awareness of women's about danger signs of pregnancy, important place to focus to initiate cascade of improvement in poor perinatal outcome is necessary.
\end{abstract}

Keywords: Perinatal, Mortality, Neonatal, Intensive, Care and Jimma Medical Center

\section{Introduction}

Deaths of human being during the perinatal period despite many measures undertaken by the world remained among the top in the list of the most important public health problems.

Worldwide, there are over 6.3 million perinatal deaths a year, almost all of which occur in developing countries, and $27 \%$ of them in the least developed countries alone. Stillbirths account for over half of all perinatal deaths. One third of stillbirths takes place during delivery, and are largely avoidable. Intrapartum deaths (i.e. those occurring during delivery) are closely linked to place of, and care at, delivery [19].

In Sub Saharan Africa approximately 30 million women become pregnant in a year. Of those, about 1 million deliveries are stillborn; at least 1 million babies die in their first month of life and 0.5 million die on the first day. About 4 million LBW babies and others with neonatal complications may live but not reach their full potential. Africa accounts for $11 \%$ of world's population but more than $25 \%$ of the worlds' new born deaths. 
According to the retrospective review of perinatal deaths that occurred during 1990-1999 at Jimma University Teaching Hospital, the overall perinatal mortality rate during the study period was 138.9 per thousand live births [18]. Recently, as per retrospective review of data derived from monthly perinatal mortality report over 30 months at JUTH [29] the overall PMR was found to be 109.3/1000 total births and the adjusted PMR 103/1000 live births. During the study period there were a total of 9585 mothers who gave birth to a total of 9,921 births at JUTH of which 1,084 were perinatal deaths (852 stillbirths \& 232 ENNDs).

The main primary obstetric cause of perinatal deaths was mechanical factors followed by; APH, HDP with or without APH and congenital anomaly in decreasing order. according to this analysis there is a statistically significant association of ENND with maternal age of 20-24 years, prim parity, ANC, preterm delivery, and weight at birth of $<2500 \mathrm{Gms}$. [29].

This study was conducted to assess the overall perinatal mortality rate and determine the primary obstetric causes and factors associated with perinatal deaths in the study area and period so as to establish a foundation of knowledge and understanding of perinatal death.

According to world health organization of perinatal and neonatal mortality report 2000, country, global and regional estimate every year, more than 7 million perinatal deaths occur across the globe (3.5 million stillbirths and 4 million neonatal deaths), which is higher than the combined annual all age level deaths due to AIDS (2.1 million), tuberculosis (1.6 million) and malaria (1.3 million) (2-6). About $99 \%$ of these perinatal deaths occur in low and middle income countries [19, 29].

Perinatal mortality rate and associated factors are tried to address in different countries including Africans. But, the decrement in the magnitude of still birth and neonatal mortality is not significant in underdeveloped countries particularly Ethiopia. Globally, declines have been observed in infant and child mortality as a result of public health interventions such as immunization and treatment for infectious diseases. However, progress towards reducing perinatal deaths has been slow and negligible. Every year, more than 7 million perinatal deaths occur across the globe (3.5 million stillbirths and 4 million neonatal deaths). About $99 \%$ of these perinatal deaths occur in low and middle income countries $[8,9]$. Unfortunately, approximately half of these perinatal deaths usually occur at home, unnamed and unrecorded, and thus unaccounted for [9]. In Ethiopia In 2006, the PMR estimated by WHO was $57 / 1000$ total births with about 2:1 ENNDs to stillbirths ratio [8]. Because of the high magnitude of the problem and its direct linkage with the quality of health service during pregnancy, per partum and in the first month of the neonates' life, PMR and neonatal mortality rate (NMR) are used as an important indicators of the health status of a country [8,35]. Furthermore, neonatal mortality is the major contributor to infant mortality rate (IMR), which was identified as one of the UN millennium development goal indicators (MDG 4). Previous report has shown that for every maternal death, there are an estimated 10 perinatal deaths [36]. Furthermore, because of the strong linkage of perinatal deaths with maternal deaths, about twothirds of the causes.

So this research is aimed at determining the perinatal mortality and associated factors for mothers admitted for termination of pregnancy complicated with IUFD or those encountered intrapartum fetal death while laboring in JUMC labor ward as well as those encountered neonatal death after delivering in this labor ward.

A case control study for perinatal mortality was conducted in Hawassa University hospital between 2008 and 2010. A total of 1356 newborns (452 cases and 904 controls) were included in this analysis. The adjusted perinatal mortality rate was $85 / 1000$ total delivery. Stillbirths accounted for $87 \%$ of total perinatal mortality. The proportion of hospital perinatal deaths was $26 \%$. Obstructed labor was responsible for more than one third of perinatal deaths. Adjusted odds ratios revealed that obstructed labor, malpresentation, preterm birth, antepartum hemorrhage and hypertensive disorders of pregnancy were independent predictors for high perinatal mortality. In the subgroup analysis, among others, obstructed labor and antepartum hemorrhage found to have independent association with both stillbirths and early neonatal deaths [30].

Data for all consecutive deliveries in the labor ward complex of Lagos University Teaching Hospital (LUTH) between June 2002 and November 2002 were obtained from the patients' record and by interviewing the mothers using a questionnaire. The babies were followed up for 7 days postdelivery. There were $51(8.5 \%)$ perinatal deaths made up of $43(7.1 \%)$ stillbirths (15 fresh and 28 macerated) and $8(6.1 \%)$ early neonatal deaths giving a perinatal mortality rate of $84.6 / 1000$. Maternal factors that significantly affected perinatal deaths were maternal age, parity, antenatal care booking and the hospital where the mother was booked for antenatal care, number of previous child deaths, and complications of pregnancy. Mode of delivery and complications of labor were the significant intrapartum factors. Fetal factors that influenced perinatal deaths were fetal presentation, birth weight, and Apgar scores at 1 and 5 min. When multiple logistic regression (multivariable analysis) of perinatal mortality on possible risk factors was done, only the Apgar score at $5 \mathrm{~min}$, birth weight, and parity were significant risk factors [31].

The study done on secondary analysis of the South African Perinatal Problems Identification Program (PPIP) database for the Province of Mpumalanga was undertaken for the period October 2013 to January 2014, inclusive. The studu indicated that; there were 23503 births and 687 late perinatal deaths (stillbirths of $\geq 1000 \mathrm{gr}$ or $\geq 28$ weeks gestation and early neonatal deaths up to day 7 of neonatal life) in the study period. The rate of maternal complication in macerated stillbirths, fresh stillbirths and early neonatal deaths was $50.4 \%, 50.7 \%$ and $25.8 \%$ respectively. Mothers on the other late perinatal deaths were healthy. Maternal hypertension and obstetric hemorrhage were more likely in still births ( $\mathrm{p}=$ 
$<0.01$ for both conditions), whereas ENNDs were more likely to have a healthy mother $(p<0.01)$. The main causes of neonatal death were related to immaturity $(48.7 \%)$ and hypoxia (40.6\%). $173(25.2 \%)$ of all late perinatal deaths had a birth weight less than the 10th centile for gestational age [32].

One year study from 1st January to 31 st December 2001 was carried out in the Department of Obstetrics and Gynecology, Jinnah Postgraduate Medical Centre, Karachi. During the one year period from 1st January to $31 \mathrm{st}$ December, 2001, there were 7743 deliveries and 753 perinatal deaths. Five hundred and sixty nine were still born and 184 died within 7-days of birth. The perinatal mortality rate (PNMR) was 97.2/1000 total leading cause of stillbirth was hypertensive disease of mother in 180 (24\%). This included Pregnancy Induced Hypertension (PIH) 106 (14\%) and eclampsia 74 (10\%). The next common cause was mechanical, accounted for 161 (21.4\%). Ante partum hemorrhage (APH) was responsible for $151(20 \%)$ perinatal deaths and low birth weight (LBW) was identified in $108(14.4 \%)$. Congenital malformation caused deaths in 47 (6.2\%), maternal medical disorders as jaundice, anemia and diabetes in 24 (3.2\%) and neonatal infections as Respiratory Distress Syndrome (RDS), probable pneumonia, bleeding disorders and septicemia caused deaths in 35 (4.8\%) [33].

According to research conducted through a hospital-based surveillance and case-control study, in Ahmedabad, India from July 1987 to June, 1988, the perinatal mortality rate was found to be 79.0 per 1000 , and was highest for preterm lowbirth-weight babies. The case-control study of 451 stillbirths, 160 early neonatal deaths and 1465 controls showed that poor maternal nutritional status, absence of antenatal care, and complications during labor were independently associated with substantially increased risks of perinatal death. Multivariate analyses indicate that socioeconomic factors largely operate through these proximate factors and do not have an independent effect. Estimates of attributable risk derived from the prevalence of exposures in the population survey suggest that improvements in maternal nutrition and antenatal and intrapartum care could result in marked reductions of perinatal mortality [34].

Recently retrospective review of perinatal deaths was conducted at our hospital over two and half years from September 2012 to February 2015 revealing the overall perinatal mortality to be $109.3 / 1000$ total births with stillbirths to early neonatal death ratio of 3.6:1. Data on maternal socio-demographic, pregnancy characteristics and clinical conditions pertaining to the perinatal outcomes were obtained from monthly perinatal mortality audit report with the objectives of assessing the overall perinatal mortality rate and associated factors. Most of the mothers were; 25-29 years old, para II-IV and had ANC accounting for 30.6\%, $60.3 \%$ and $73.8 \%$ respectively. More than three-fourth of the cases were delivered at term and close to two third of them weighted 2500-3999 Gms at delivery. They also found that perinatal mortality has an association with maternal parity,
ANC, weight and gestational age at delivery. Mechanical factors followed by antepartum hemorrhage, hypertensive disorder of pregnancy and congenital anomalies were primary obstetric causes initiating the cascade of perinatal death [28].

Perinatal mortality is high in developing countries and deaths during the perinatal period have become a more important public health problem. Perinatal mortality is one of the most sensitive indices of maternal and child health. As a result, conducting a research on this area is the most important way to address the factors that lead to this perinatal mortality.

This study enables health managers and health professionals to use for planning the necessary budget and improve the quality of care. It is also used as base line data do further research on the area and give clue for other researcher.

\section{Methods}

\subsection{Study Area and Period}

The study were conducted in Oromia Region, Jimma Zone, Jimma Town, at Jimma University Specialized Hospital, from Jan 1, 2019-April 30, 2019. Jimma town is located at $335 \mathrm{~km}$ south west of Addis Ababa with estimated total population of 120,960 of which males account 60,824 and females account 60,136 according to 2007 census. Its weather condition is woinadega and its annual average rainfall is $1200 \mathrm{~mm}$ and it is located greater than $1400 \mathrm{~m}$ attitude above sea level. In the city there are many different types of governmental, nongovernmental \& private organization.

Study Design

Retrospective study design was used as the study design.

\subsection{Source Population}

All neonates admitted to neonatal intensive care unit were considered as the source of population.

\subsubsection{Study Population}

All neonates admitted to neonatal intensive care unit, from Jan 1, 2019 to April 30, 2019 were used as target population.

\subsubsection{Inclusion Criteria}

All neonates admitted to neonatal intensive care from Jan 1, 2019 to April 30, 2019 with complete records were included to the study.

\subsubsection{Exclusion Criteria}

Neonates admitted to neonatal intensive care with incomplete records were excluded from the study.

Sample size and sampling technique

All neonates admitted to neonatal intensive care from Jan 1, 2019 to April 30, 2019 with complete records. There are 190 neonate admitted to NICU during the study period out this only 186 have complete information which is considered as sample size of the study. 


\subsection{Study Variables}

\subsubsection{Independent Variables}

1. Socio-demographic characteristics: sex of neonate.

2. Obstetrics: labor stage, gestation age, maternal underlying disease (hypertension, cardiac disease).

3. Prenatal: birth weight, maceration (grade), Apgar score, major congenital anomalies.

4. Maternal and prenatal outcomes: mode of delivery $(\mathrm{C} / \mathrm{S}$, vaginal, instrumental, destructive deliveries).

\subsubsection{Dependent Variable}

Neonatal mortality was considered as a dependent variable.

\subsection{Data Collection Procedure}

Data was collected by trained data collectors using a check list sheet from the medical records of those neonates who had been admitted to the NICU over the specified time period.

\subsection{Data Analysis Plan}

Data was edited and entered into SPSS version 21 for cleaning and analysis. Data was explored first to clean (check for outliers, missing values and normality) using descriptive statistics. Descriptive statistics such as percentages and frequency counts were produced.

Magnitude of perinatal mortality was determined after the end of the study for newborns. Cross-tabulation result was conducted and simple relationship was checked between dependent and independent variables. Variables with p-value less than 0.05 were considered as independent predictors of outcome variable.

\section{Results}

Socio-demographic characteristics.

There was total of 191 neonate admitted to NICU and 186 of them had complete information. The response rate was 97.4\%.

This study revealed that perinatal mortality was 55 $(29.6 \%)$. Regarding sex; majority of new born were male 117 $(62.9 \%)$.

Finding of this study also showed that majority 156 $(83.9 \%)$ of new born were delivered by spontaneous vaginal delivery while $30(16.1 \%)$ of deliveries were by cesarean section (Table 1).

Table 1. Socio-demographic characteristics, Place and mode of delivery at JUMC, Southwest Ethiopia, April 10 to April 25, 2019.

\begin{tabular}{llll}
\hline Variables & Category & Frequency & Percent (\%) \\
\hline \multirow{2}{*}{ Sex } & Male & 117 & 62.90 \\
& Female & 69 & 37.10 \\
Mode of delivery & Cesarean section & 30 & 16.1 \\
& SVD & 156 & 83.9 \\
Place of delivery & Home delivery & 7 & 3.8 \\
& Same Facility & 57 & 30.6 \\
\hline
\end{tabular}

Admission Information indicates.

This study revealed that average admission weight of the new born is $2513+841$ gram while three of the newborn weight is unknown while their mean birth weight is $2445+843$ from 111 new born. The average respiratory rate (RR) and apical heart rate (AHR) per one minute were
$55.2+15.36$ and $137.45+14.31$ respectively. The minimum and maximum gestational age was 27 and 41 weeks respectively. First and fifth mint APGAR score were 3/4 and 9/10 from 38 new born only, the rest have unknown APGAR score.

Table 2. Descriptive Statistics for Admission Information at JUMC April 10 to April 25, 2019.

\begin{tabular}{llll}
\hline Variables & N & Mean & Std. Deviation \\
\hline Admission Weight (gm) & 183 & 2513.19 & 841.75 \\
Temperature $\left({ }^{\circ} \mathrm{C}\right)$ & 186 & 36.63 & 1.13 \\
Respiratory Rate (RR) per one minute & 186 & 55.20 & 15.36 \\
Apical Heart Rate (AHR) per one minute & 185 & 137.45 & 36.04 \\
Gestational Age (in weeks) & 164 & 5.97 & 3.06 \\
APGAR Score 1'(At birth) & 38 & 7.63 & 1.53 \\
APGAR Score 5' (At birth) & 38 & 2445.75 & 1.62 \\
Birth weight (gm) & 111 & 843.09 \\
\hline
\end{tabular}

Cause of Admission

Findings of this study indicated that regarding the cause of admission to NICU Prematurity, LBW, Sepsis, Respiratory Distress syndrome a accounts $74.7 \%, 77.1 \%, 78.3 \%$ and $473.8 \%$ respectively while perinatal asphyxia (PNA) and congenital malformation account $49.3 \%$ and $38.7 \%$ (Table 3 ). 
Table 3. Admission cause at JUMC, Southwest Ethiopia, April 10 to April 25, 2019.

\begin{tabular}{|c|c|c|c|c|}
\hline \multirow{2}{*}{ Variables } & \multicolumn{2}{|l|}{ Yes } & \multicolumn{2}{|l|}{ No } \\
\hline & Frequency & Percent & Frequency & Percent \\
\hline Prematurity & 71 & $74.7 \%$ & 24 & $25.3 \%$ \\
\hline LBW (low birth weight) & 74 & $77.1 \%$ & 22 & $22.9 \%$ \\
\hline Respiratory Distress syndrome & 76 & $73.8 \%$ & 27 & $26.2 \%$ \\
\hline Perinatal Asphyxia (PNA) & 34 & $49.3 \%$ & 35 & $50.7 \%$ \\
\hline Congenital Malformation & 24 & $38.7 \%$ & 38 & $61.3 \%$ \\
\hline
\end{tabular}

Managements in Neonate Intensive Care Unit

Majority of $94 \%$ new born gets glucose, $93.9 \%$ gets Antibiotics, 90.6\% were managed using Incubator thermal care while $75.9 \%$ gets oxygen and $71.6 \%$ uses Resuscitation.
Moreover, Continuous Positive Air way Pressure CPAP, Phototherapy, Anticonvulsants and Blood Transfusion were managed for $49.2 \%, 38.6 \%, 29.6 \%$ and $24.6 \%$ (Table 4 ).

Table 4. Managements at Neonate Intensive Care Unit, JUMC, April 10 to April 25, 2019

\begin{tabular}{lllll}
\hline \multirow{2}{*}{ Variables } & Yes & & No & Percent \\
\cline { 2 - 5 } & Frequency & Percent & Frequency & $50.8 \%$ \\
\hline Continuous Positive Air way Pressure CPAP & 32 & $49.2 \%$ & 33 & $28.4 \%$ \\
Resuscitation & 58 & $71.6 \%$ & 23 & $6.1 \%$ \\
Antibiotics & 154 & $93.9 \%$ & 10 & $70.4 \%$ \\
Anticonvulsants & 21 & $29.6 \%$ & 50 & $61.4 \%$ \\
Phototherapy & 27 & $38.6 \%$ & 43 & $6.0 \%$ \\
Glucose & 156 & $94.0 \%$ & 10 & $24.1 \%$ \\
$\mathrm{O}_{2}$ (oxygen) & 101 & $75.9 \%$ & 32 & $75.4 \%$ \\
Blood Transfusion & 17 & $24.6 \%$ & 52 & $9.4 \%$ \\
Incubator thermal care & 135 & $90.6 \%$ & 14 & \\
\hline
\end{tabular}

The average age of death was $4.5+3.7$ days with the minimum 1 and maximum 20 days of life. The mean length of hospital stay and weight at discharge were 6.2+4.2 and 2539.8+885.5 gram respectively (table 5).

Table 5. Average Weight, age and length of hospital stay.

\begin{tabular}{llllll}
\hline Descriptive Statistics & & & & & \\
\hline & N & Minimum & Maximum & Mean & Std. Deviation \\
\hline Age in Days for Died & 54 & 1.00 & 20.00 & 4.5000 & 3.72016 \\
Length of Hospital Stay & 173 & 1.00 & 30.00 & 6.2139 & 4.20623 \\
Weight at Discharge & 148 & 770.00 & 4850.00 & 2539.8108 & 885.58283 \\
\hline
\end{tabular}

Cause of Death among admitted to Cause of Death among those admitted to NICU

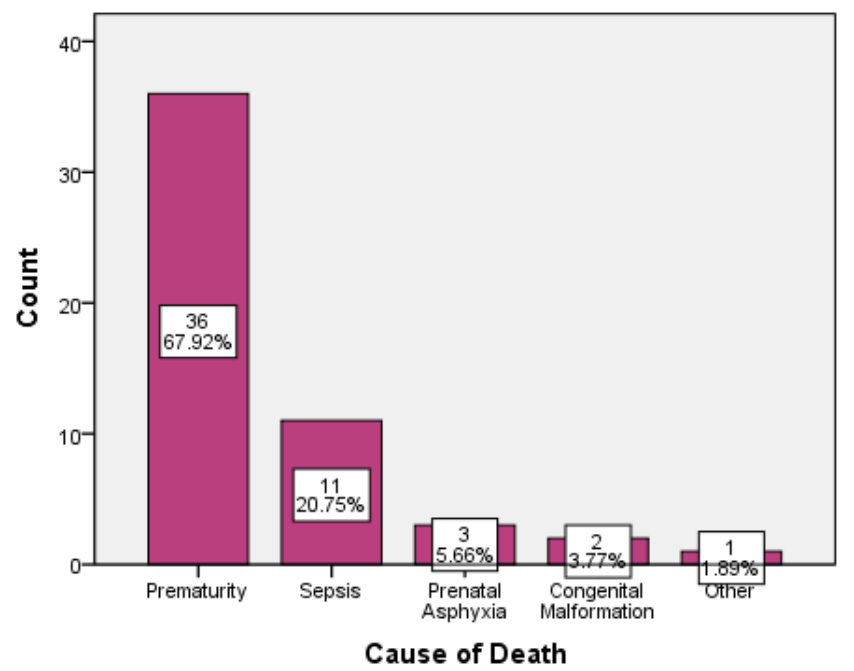

Figure 1. Cause of Death at JUMC, Southwest Ethiopia, April 10 to April $25,2019$.
The cause of death among those admitted to NICU the most $67.9 \%$ are prematurity, next $20.75 \%$ Sepsis, $5.66 \%$ Prenatal Asphyxia and $3.77 \%$ is due to Congenital malformation while only one infants were died because of other factors (Figure 1).

Factors associated with the Neonatal mortality among the study subjects.

Association between neonatal outcomes with Admission information.

The association of Neonatal outcome at Discharge to different variables (Sex, Mode of delivery, Place of delivery, Prematurity, LBW (low birth weight), Sepsis, Respiratory Distress syndrome, Perinatal Asphyxia (PNA) and Congenital Malformation) were assessed using chi-square statistical tools. The result indicated in revealed that there was statistically significant association between the Neonatal outcome at Discharge with Prematurity, LBW (low birth weight), Respiratory Distress syndrome and Congenital malformation while there is no association with sex, mode of delivery write code, place of delivery, sepsis and perinatal asphyxia (PNA) at 5\% level of significance [6]. 
Table 6. Association between admission problem and information with neonatal outcome discharge at JUMC, April 10 to April 25 , 2019.

\begin{tabular}{|c|c|c|c|c|}
\hline \multirow{2}{*}{ Admission Problem and information } & \multirow{2}{*}{ Category } & \multicolumn{2}{|c|}{ Neonatal Outcome at Discharge } & \multirow{2}{*}{$X^{2}(p)$ Value } \\
\hline & & Recovered & Died & \\
\hline \multirow{2}{*}{ Sex } & Male & $85(72.6 \%)$ & $32(27.4 \%)$ & \multirow{2}{*}{$0.75(0.38)$} \\
\hline & Female & $46(66.7 \%)$ & $23(33.3 \%)$ & \\
\hline \multirow{3}{*}{ Mode of delivery write code } & $\mathrm{C} / \mathrm{S}$ & $24(80.0 \%)$ & $6(20.0 \%)$ & \multirow{2}{*}{$1.57(0.21)$} \\
\hline & SVD & $107(68.6 \%)$ & $49(31.4 \%)$ & \\
\hline & Home delivery & $5(71.4 \%)$ & $2(28.6 \%)$ & \multirow{3}{*}{$0.005(0.997$} \\
\hline \multirow[t]{2}{*}{ Place of delivery } & Same Facility & $40(70.2 \%)$ & $17(29.8 \%)$ & \\
\hline & Referred & $86(70.5 \%)$ & $36(29.5 \%)$ & \\
\hline \multirow{2}{*}{ Prematurity } & Yes & $35(49.3 \%)$ & $36(50.7 \%)$ & \multirow{2}{*}{$10.82(0.001)$} \\
\hline & No & $21(87.5 \%)$ & $3(12.5 \%)$ & \\
\hline \multirow{2}{*}{ LBW (low birth weight) } & Yes & $38(51.4 \%)$ & $36(48.6 \%)$ & \multirow{2}{*}{$8.62(0.003)$} \\
\hline & No & $19(86.4 \%)$ & $3(13.6 \%)$ & \\
\hline \multirow{2}{*}{ Sepsis } & Yes & $64(68.1 \%)$ & $30(31.9 \%)$ & \multirow{2}{*}{$0.39(0.53)$} \\
\hline & No & $16(61.5 \%)$ & $10(38.5 \%)$ & \\
\hline \multirow{2}{*}{ Respiratory Distress syndrome } & Yes & $38(50.0 \%)$ & $38(50.0 \%)$ & \multirow{2}{*}{$8.12(0.004)$} \\
\hline & No & $22(81.5 \%)$ & $5(18.5 \%)$ & \\
\hline \multirow{2}{*}{ Perinatal Asphyxia (PNA) } & Yes & $16(47.1 \%)$ & $18(52.9 \%)$ & \multirow{2}{*}{$3.28(0.07)$} \\
\hline & No & $24(68.6 \%)$ & $11(31.4 \%)$ & \\
\hline \multirow{2}{*}{ Congenital Malformation } & Yes & $19(79.2 \%)$ & $5(20.8 \%)$ & \multirow{2}{*}{$3.67(0.055)$} \\
\hline & No & $21(55.3 \%)$ & $17(44.7 \%)$ & \\
\hline
\end{tabular}

Association between neonatal outcomes with Managements factor

The association of Neonatal outcome at Discharge to management factors (Continuous Positive Air way Pressure CPAP, Resuscitation, Prolonged skin to skin thermal care (KMC), Antibiotics, Anticonvulsants, Phototherapy, Glucose, $\mathrm{O}_{2}$ (oxygen), Blood Transfusion) and Incubator thermal care) were assessed using chi-square statistical tools. The result indicated in (Table 7) revealed that there was statistically significant association between the Neonatal outcome at Discharge with Continuous Positive Air way Pressure CPAP, Resuscitation, Prolonged skin to skin thermal care (KMC) and Incubator thermal care. While there is no association with Antibiotics, Anticonvulsants, Phototherapy, Glucose, $\mathrm{O}_{2}$ (oxygen) and Blood Transfusion at 5\% level of significance.

Table 7. Association between Managements factor with neonatal Outcome at Discharge, JUMC, April 10 to April 25,2019

\begin{tabular}{|c|c|c|c|c|}
\hline \multirow{2}{*}{ Variables } & \multirow{2}{*}{ Category } & \multicolumn{2}{|c|}{ Outcome at Discharge } & \multirow{2}{*}{$X^{2}$ (p) Value } \\
\hline & & Recovered & Died & \\
\hline \multirow{2}{*}{ Continuous Positive Air way Pressure CPAP } & Yes & $9(28.1 \%)$ & $23(71.9 \%)$ & \multirow{2}{*}{$11.23(0.001)$} \\
\hline & No & $23(69.7 \%)$ & $10(30.3 \%)$ & \\
\hline \multirow{2}{*}{ Resuscitation } & Yes & $11(19.0 \%)$ & $47(81.0 \%)$ & \multirow{2}{*}{$36.06(0.000)$} \\
\hline & No & $21(91.3 \%)$ & $2(8.7 \%)$ & \\
\hline \multirow{2}{*}{ Prolonged skin to skin thermal care (KMC) } & Yes & $54(83.1 \%)$ & $11(16.9 \%)$ & \multirow{2}{*}{$3.91(0.021)$} \\
\hline & No & $9(56.3 \%)$ & $7(43.8 \%)$ & \\
\hline Antibiotics & No & $9(90.0 \%)$ & $1(10.0 \%)$ & $2.43(0.12)$ \\
\hline \multirow{2}{*}{ Anticonvulsants } & Yes & $11(52.4 \%)$ & $10(47.6 \%)$ & \multirow{2}{*}{$0.57(0.45)$} \\
\hline & No & $31(62.0 \%)$ & $19(38.0 \%)$ & \\
\hline \multirow{2}{*}{ Phototherapy } & Yes & $19(70.4 \%)$ & $8(29.6 \%)$ & \multirow{2}{*}{$0.71(0.4)$} \\
\hline & No & $26(60.5 \%)$ & $17(39.5 \%)$ & \\
\hline \multirow{2}{*}{ Glucose } & Yes & $105(67.3 \%)$ & $51(32.7 \%)$ & \multirow{2}{*}{$0.22(0.64)$} \\
\hline & No & $6(60.0 \%)$ & $4(40.0 \%)$ & \\
\hline $\mathrm{O}_{2}$ (oxygen) & No & $23(71.9 \%)$ & $9(28.1 \%)$ & $2.13(0.14)$ \\
\hline \multirow{2}{*}{ Blood Transfusion) } & Yes & $9(52.9 \%)$ & $8(47.1 \%)$ & \multirow{2}{*}{$0.23(0.63)$} \\
\hline & No & $31(59.6 \%)$ & $21(40.4 \%)$ & \\
\hline \multirow{2}{*}{ Incubator thermal care } & Yes & $88(65.2 \%)$ & $47(34.8 \%)$ & \multirow{2}{*}{$5.58(0.018)$} \\
\hline & No & $13(92.9 \%)$ & $1(7.1 \%)$ & \\
\hline
\end{tabular}

\section{Discussion}

Care provided during per partum period especially during labor is the most important thing to give optimum care as far as improvement in perinatal outcome is considered.

According to this study finding there was high perinatal mortality which was 55 (29.6\%) perinatal deaths per 1000 total deliveries. Of total perinatal mortality $67.9 \%, 20.75 \%, 5.66 \%$ and $3.77 \%$ were due to prematurity, Sepsis, Prenatal Asphyxia and Congenital malformation respectively. This study finding is lower than multicenter facility based study of perinatal mortality conducted by WHO from 2001-2004 in sub-Saharan Africa which was found to be $12.5 / 1000$ births. It is almost two times of the national magnitude as it was estimated by WHO [14]. This finding is higher when compared with the 
study finding done in Tanzania were Birth asphyxia was found to be the significant cause of perinatal mortality [22]. PNM at Muhimbili National Hospital, Dares Salaam, Tanzania was 92/1000 total births with intrapartum causes of fresh stillbirth and ENND being birth asphyxia (47.4\% among fresh still births and $51.3 \%$ among the ENND).

The reason for higher perinatal mortality in this hospital might be due to its being the only referral hospital at tertiary level at southwestern part of the country. As there is inadequate laboratory investigation and no ultrasound imaging availability at health center generally and some primary hospitals they are also referred for diagnosis establishment and confirmation.

This study also revealed that congenital anomalies which are incompatible with life are diagnosed intrapartum and responsible for $10 \%$ of total perinatal mortalities. This finding also supports the above ideas. This all reason and others are the cause for stagnant decrement in perinatal mortality and higher magnitude.

This all contributed for high perinatal mortality in our setup. By improving referral system provided that timely detection of complication and referral made sure at least we can save babies with normal birth weight dying from easily avoidable causes especially mechanical cause as it 's burden is shown above [19, 35, 36].

According to a prospective cohort study carried out among a cohort of neonates delivered in seven hospitals of Tigray from April to July, 2014 and followed up for a total of 28 days, neonatal complications are also one of the independent predictors for NM. The main causes of neonatal complications being meconium aspiration, prematurity, low birth weight and prolonged labor, which lead to Apgar score less than 7 and birth asphyxia [17]. So, it is similar with the current study.

\section{Conclusion}

The magnitude of neonatal death as it is revealed by this study was unacceptably high. Most of the neonates were male. The highest cause of admission to neonatal intensive care unit was Sepsis followed by low birth weight and prematurity while the least was perinatal asphyxia and congenital malformation. For early neonatal deaths prematurity and sepsis were the leading cause of death next to prenatal asphyxia and congenital malformation.

Majority of the neonates were managed by glucose, antibiotics and using incubator thermal care while some gets oxygen and resuscitation. In contrast, few of the infants were managed by continuous positive air way pressure (CPAP), phototherapy, anticonvulsants and blood transfusion.

\section{Recommendation}

Based on the study findings and objectives of the study, these recommendations have been made at different levels.

1. Most of the causes and contributory factors for perinatal death as is well described above are easily manageable if attention is given to quality antenatal care, timely detection of complications and improving referral procedures based on particular complication.

2. Ultrasound examination should be provided to pregnant mothers at least once during pregnancy so that congenital anomalies that are incompatible with life will be detected and managed early.

3. Health care providers should also teach on danger sign of pregnancy by using antenatal care visit as special opportunity to build the clients' understanding and awareness.

4. Intrapartum care should be as optimum as possible so that early detection of problems and managing them accordingly will be optimized in order to improve perinatal outcome.

\section{Authors' Contribution}

Gebeye Muleta conceived and designed the protocol. A Gebeye Muleta, contributed on data analysis, and checked the draft. Abiru Neme and Gadisa Bekele prepared manuscript. Abiru Neme and Gadisa Bekele read and approved the final paper.

\section{Conflict of Interests}

All authors declared that they have no conflict of interests. Jimma University covered only the survey cost for this study and there is no any funding organization.

\section{Acknowledgements}

We would like to thank the Jimma Medical Center administrative as well as the administrative staffs for all the help and support given for us during the data collection period. We also thank Jimma university institute of health for permitting me to conduct this study.

\section{References}

[1] Stanton C, Lawn JE, Rahman H, Wilczynska-Ketende K, Hill K. Stillbirth rates: delivering estimates in 190 countries. Lancet. 2006; 367: 1487-1494.

[2] Macdorman MF, Munson ML, Kirmeyer S. Fetal and perinatal mortality, United States, 2004. Natl Vital Stat Rep. 2007; 56: $1-19$.

[3] Thomson, A. M. \& Barron, S. L. Perinatal mortality. In: Barron, S. L. \& Thomson, A. M., ed. Obstetrical epidemiology. London, Academic Press, 1983, pp. 346-398.

[4] Chamberlain, G. Better perinatal health: background to perinatal health. Lancet, 2: 1061-1063 (1979).

[5] World Summit for Children in 1990, the United Nations Millennium Declaration and the United Nations Special Session on Children in 2002. 
[6] World Health Organization (2010) International statistical classification of diseases and related health problems. Geneva, Switzerland.

[7] World Health Organization, UNICEF, UNFPA and The World Bank, (2012): Trends in maternal mortality 1990-2010.

[8] WHO Perinatal and Neonatal Mortality for the Year 2000: Country, Regional and Global Estimates. WHO; Geneva: 2006.

[9] World Health Organization (2006) Neonatal and perinatal mortality: Country, Regional \& Global estimates. Geneva, Switzerland. Regional Committee for Africa, 58 [2011-0613].

[10] Bakketeig, L. S. et al. Perinatal mortality. In: Bracken, M., ed. Perinatal epidemiology. New York, Oxford University Press, 1984, pp. 99-151.

[11] Watts, T. \& Harris, and R. R. A case-control study of stillbirths at a teaching hospital in Zambia, 1979-80: antenatal factors. Bulletin of the World Health Organization, 60: 971979 (1982).

[12] Barros, C. et al. Perinatal mortality in southern Brazil: a population-based study of 7392 births. Bulletin of the World Health Organization, 65: 95-105 (1987).

[13] Ferraz, E. M. \& Gray, R. H. A case-control study of stillbirths in North-east Brazil. International journal of gynaecology and obstetrics, 34: 13-19 (1991).

[14] Gray, R. H. et al. Determinants of early neonatal mortality, Natal, North-east Brazil: results of a surveillance and casecontrol study. International journal of epidemiology, (In press).

[15] World Health Organization (2006) Neonatal and perinatal mortality: Country, Regional \& Global estimates. Geneva, Switzerland, 33: 18-20 (2006).

[16] Yifru B, Asres B (2014) prenatal mortality trends in Ethiopia. Ethiop J Health Sci 24: 29-40.

[17] Hayelom Gebrekirstos Mengesha, Alem Desta Wuneh, Wondwossen Terefe Lerebo, Tesfay Hailu Tekle (2016) Survival of neonates and predictors of their mortality in Tigray region, Northern Ethiopia: prospective cohort study. BMC Pregnancy and Childbirth 16: 1.

[18] Liu L, Johnson HL, Cousens S, Perin J, Scott S, et al. (2012) Child Health Epidemiology Reference Group of WHO and UNICEF; Global, regional, and national causes of child mortality: an updated systematic analysis for 2010 with time trends since 2000. Lancet 379: 2151-2161.

[19] Gibb DM, Kizito H, Russell EC, Chidziva E, Zalwango E, Nalumenya R, et al. (2012) Pregnancy and Infant Outcomes among HIV-Infected Women Taking Long-Term ART with and without Tenofovir in the DART Trial. PLoS Med 9 (5): e1001217.

[20] Chinkhumba J, De Allegri M, Muula AS, Robberstad B (2014) Maternal and perinatal mortality by place of delivery in sub-Saharan Africa: a meta-analysis of population-based cohort studies. BMC Public Health 14: 1014.

[21] Ngoc NT, Merialdi M, Abdel-Aleem H, Carroli G, Purwar M, et al. (2006) Causes of stillbirths and early neonatal deaths: data from 7993 pregnancies in six developing countries. Bull World Health Organ 84: 699-705.

[22] Kidanto HL, Mogren I, Roosmalen J, Thomas AN, Massawe SN, et al. (2009) Introduction of a qualitative perinatal audit at Muhimbili National Hospital, Dares Salaam, Tanzania. BMC Pregnancy and Childbirth 9: 45.

[23] Nakibuuka VK, Okong P, Waiswa P, Byaruhanga RN (2012) Perinatal death audits in a peri-urban hospital in Kampala, Uganda. Afr Health Sci 435-442.

[24] MacDorman MF, Gregory ECW (2015) Fetal and perinatal mortality: United States, 2013. National vital statistics system. 64: 1-24.

[25] Allanson ER, Muller M, Pattinson RC. Causes of perinatal mortality and associated maternal complications in a South African province: challenges in predicting poor outcomes. BMC Pregnancy Childbirth. 2015; 15 (1): 37.

[26] Naeye RL, Tafari N, Marboe C, Judge DM (1977) Causes of perinatal mortality in an African city. Bull. World health organ 55: 63-65.

[27] Central Statistical Agency, ICF International (2012) Ethiopia demographic and health survey 2011. Addis Ababa, Ethiopia and Calverton, Maryland, USA.

[28] Gaym A (2000) Perinatal mortality audit at Jimma hospital, South- Western Ethiopia, 1990-1999. Ethiop J Health Dev 14: 335-343.

[29] Asefa D, Akessa GM, Araya F, Amenu D, Girma W, et al. (2016) Pattern of Perinatal Mortality among Deliveries at Jimma University Teaching Hospital, South-West Ethiopia. J Women's Health, Issues Care 5: 6.

[30] Yifru Berhan, Getachew Bayou: case control study of perinatal mortality and associated factors in Awassa university hospital, 2008-2010.

[31] ENEkure, VC Ezeaka, E Iroha, MTC Egri-Okwaji: Prospective audit of perinatal mortality among newborn babies in a tertiary health center in Lagos, Department of Paediatrics, Lagos University Teaching Hospital, Lagos, Nigeria.

[32] Emma R. Allanton, Mari Muller and Robert Pantinson Causes of perinatal mortality and associated maternal complications in a South African province: challenges in predicting poor outcomes.

[33] RaziaKorejo, ShereenBhutta, Khurshid J. Noorani, Zulfiqar A. Bhutta: An audit and trendsBhutta: An audit and trends of prenatal mortality at Jinnah post graduate medical.

[34] D. V. Mavalankar, C. R. Trivedi, \& R. H. Gray Levels and risk factors for perinatal mortality in Ahmedabad, India.

[35] Richardus JH, Richardus JH, Graafmans WC, VerlooveVanhorick SP, Mackenbach JP. The perinatal mortality rate as an indicator ofquality of care in international comparisons. Med Care 1998; 36 (1): 54-66. 36.

[36] Campbell O, Koblinsky M, Taylor P. Off to a rapid start: appraising maternal mortality and services. Int $J$ Gynecol Obstet 1995; 48 (Suppl): S33-S. 